\title{
COMPLESSITÀ E COSCIENZA: DALLA TEORIA AL LETTO DEL PAZIENTE
}

\author{
MARCELLO MASSIMINI (*)
}

\begin{abstract}
DEDICA. Il tono di questa relazione, che racconta di un viaggio ai confini della coscienza, è profondamente influenzato dalla mia lettura dello spirito del professor Savoldi. Non ho mai avuto il piacere di incontrare il professor Savoldi, ma bo avuto il piacere di conoscerlo leggendo "la Coscienza" [1], un saggio tanto corposo quanto denso. Perdendomi tra le pagine di questo libro, penso di essermi formato un'idea, seppur molto approssimativa e personale, dell'entusiasmo e della larghezza che banno caratterizzato lo sguardo del professor Savoldi nella sua esplorazione del mistero supremo, quello del rapporto tra materia e coscienza. Tra le molte cose che ho apprezzato, ciò che mi ha davvero colpito durante la lettura è stato l'occorrere di una forma di riconoscimento, l'emergere graduale di un senso di familiarità dovuta ad una serie di particolarissime passioni in comune. Tra queste, voglio ricordare l'attrazione per la filosofia e la neurologia e, in particolare, il potente istinto a esplorare le relazioni tra due discipline davvero distanti: la fenomenologia e l'elettroencefalografia. Per questo, più che della tipica relazione scientifica, il testo che segue ha il carattere del racconto di un viaggio, di una traiettoria in bilico tra paesaggi complessi e confini poco definiti. L'intento è quello di toccare temi che credo cari al professor Savoldi, con gli occhi dello scienziato appassionato che vede nelle onde elettriche del cervello il mare più profondo e le montagne più maestose, la bellezza della fisica e l'irriducibilità della coscienza.
\end{abstract}

SuNTO. - Tipicamente, valutiamo il livello di coscienza di altri individui basandoci sulla loro capacità di interagire con l'ambiente circostante. Tuttavia, sappiamo bene che la coscienza può essere interamente generata all'interno del cervello, in assenza di qualsiasi comunicazione con il modo esterno; ciò accade, quasi ogni notte, quando sogniamo. A causa di questa discrepanza, la presenza di coscienza può essere misconosciuta in pazienti cerebrolesi che emergono dal coma ma non comunicano. Lo sviluppo di una misura oggettiva e affidabile della capacità di coscienza rappresenta una delle grandi

(*) Università degli Studi, Milano, Italia. E-mail: marcello.massimini@unimi.it 
sfide per scienza medica. Una sfida che dobbiamo accettare e vincere in nome dei milioni di pazienti che oggi sopravvivono al coma grazie alla moderna terapia intensiva. Non solo: in un futuro non troppo lontano, potremmo trovarci circondati da macchine artificiali costruite copiando i circuiti del nostro cervello e sarà bene attrezzarci per capire se avremo creato solo un utile zombie o un entità che sente qualcosa. Secondo una teoria di recente formulazione, la coscienza di un sistema fisico dipende da uno specifico tipo di complessità, da un raro bilancio tra unità e diversità. Oggi, misure empiriche, ispirate da questa teoria, ci aiutano di individuare la presenza di coscienza anche all'interno di cervelli che sono completamente isolati dal mondo esterno. $\mathrm{Ci}$ aiuteranno, domani, a capire come fa un chilo e mezzo di materia gelatinosa a ospitare l'universo di un sogno?

$* * *$

ABSTRACT. - We normally assess consciousness in other individuals based on their ability to interact with the surrounding environment. A the same time, we all know very well that consciousness can be generated entirely within the brain, even in the absence of any interaction with the external world; this happens almost every night when we dream. Because of this fundamental discrepancy, the presence of consciousness may go undetected in brain-injured patients who emerge from coma but remain unresponsive. Developing a reliable, objective measure of the capacity for consciousness is one of the grand challenges for medical science. A challenge that we need to take on and face successfully for the sake of all the patients who survive coma through intensive care medicine. Besides, we may one day have to face the question of whether future artificial intelligence is just a useful zombie or a somehow conscious entity. According to a recently formulated theory, consciousness depends on a special kind of complexity in physical systems, on a unique balance between unity and diversity. Today, empirical measures inspired by this theory can help us in detecting a covert capacity for consciousness in patients who are otherwise completely isolated from the external world. Will they help us, one day, to understand how can a few pounds of jelly matter host the universe of a dream?

Abbiamo sviluppato teorie e telescopi per vedere stelle lontanissime, ma non abbiamo un metodo affidabile per vedere dove brilla la luce della coscienza nel mondo intorno a noi. Questo nonostante le neuroscienze abbiano fatto passi da gigante, soprattutto negli ultimi decenni. Ci siamo fatti un'idea abbastanza chiara di come complicati circuiti neuronali siano in grado di discriminare colori diversi, di coordinare movimenti complessi come la prensione di un oggetto, di codificare le memorie, di produrre le elaborate sequenze motorie che sono alla base della comunicazione verbale, e molto altro. Oggi, conosciamo talmente bene alcuni di questi meccanismi che possiamo riprodurli fedelmente nei circuiti artificiali di un calcolatore. Ogni anno, la stampa generalista, decine di libri e migliaia di articoli scientifici ci raccontano gli incredibili successi delle neuroscienze inducendoci a credere che, 
oramai, conosciamo la relazione tra mente e cervello in ogni suo minimo dettaglio: scoperte le aree cerebrali dell'empatia, quelle che fanno la differenza tra un cittadino americano che voterà per i repubblicani e uno che voterà per i democratici, quelle che spiegano perché alla fine compriamo una bevanda piuttosto che un'altra. Ma, quando ci avviciniamo al problema della coscienza rimaniamo quasi paralizzati di fronte a domande tanto semplici quanto fondamentali. Come fa la materia a ospitare un soggetto che semplicemente vede luce o buio? Perché la coscienza risiede nel cervello ma non nel fegato o nel cuore, come si riteneva in passato? Perché alcune strutture neurali (come la corteccia cerebrale) sembrano fondamentali, mentre altre (come il cervelletto) non lo sono affatto? Perché la coscienza svanisce durante il sonno, nonostante la corteccia cerebrale rimanga attiva? Come può improvvisamente riemergere, intensa e vivida, durante un sogno, mentre il cervello è disconnesso dal mondo esterno? E' un paziente che giace immobile nel letto a seguito di un grave incidente cerebrale ancora cosciente? Un pappagallo che parla? Un polpo che impara a navigare in un labirinto? Il supercomputer che ci batte regolarmente a scacchi?

Queste domande hanno implicazioni esistenziali che ognuno di noi, ciascuno a suo modo, ha scelto di contemplare almeno una volta, ma anche conseguenze etiche che non possiamo permetterci di ignorare. Esamineremo brevemente le une e le altre nei paragrafi che seguono.

\section{L'UNIVERSO SUL PALMO DELLA MANO}

Tutti sappiamo che la coscienza dipende da come funziona il cervello; basta un trauma cranico, o una dose adeguata di anestetico, perché tutto l'universo sparisca, noi stessi inclusi. Anche chi non è mai sprofondato nel buio del coma o nel nulla dell'anestesia generale può rendersi conto di quanto sia delicato il rapporto tra la coscienza e la materia che la ospita. Tutte le sere, appena ci addormentiamo, qualcosa inizia a cambiare nel funzionamento del nostro cervello e, a un certo punto, non esistiamo più. Questo è, di per sé, un fatto straordinario ma, tipicamente, non ci facciamo molto caso. Forse, non ci facciamo troppo caso perché non possiamo avere esperienza dell'incoscienza: quando c'è lei, non ci siamo noi. Per la stessa ragione, argomentava Epicuro, non dovremmo neppure preoccuparci della morte, infatti, "Il più terribile dunque dei mali, la morte, non è nulla per noi, perché quando ci siamo noi non c’è la 
morte, quando c'è la morte noi non siamo più." [2]. Forse il nulla del sonno profondo non ci impressiona più di tanto perché sappiamo che l'universo ricompare regolarmente al nostro risveglio, ogni mattina. Forse, più banalmente, diamo per scontata la coscienza così come tendiamo a dare per scontate molte cose importanti. Cose di cui non riusciamo ad apprezzare la fragilità e la grandezza per una semplice questione di prospettiva: ci siamo completamente immersi dentro.

Solo raramente, contingenze molto particolari ci mettono davanti alla dimensione reale delle cose. Sono eventi che non si dimenticano, come quelli occorsi, per esempio, agli astronauti delle missioni Apollo. Questi uomini non erano poeti e neppure filosofi, erano uomini d'azione, piloti militari e ingegneri; dopo anni di addestramento le loro menti erano sature di nozioni tecniche, di sequenze e di procedure di emergenza e, probabilmente, non se lo aspettavano proprio. E' successso tutto in una frazione di secondo, quando si sono girati e hanno visto il nostro pianeta sorgere dietro la Luna. In un istante, questi astronauti hanno guadagnato un senso del mondo cui, forse, solo l'asceta più illuminato può aspirare, alla fine di una vita di meditazioni. I membri delle missioni Apollo che sognavano la Luna hanno, in realtà, scoperto la Terra, semplicemente perché l'hanno potuta vedere da abbastanza lontano (da circa 300 mila chilometri). Le descrizioni che gli astronauti fanno di questo evento possono apparire ingenue, ma sono più che altro commoventi, perché sono genuine e spontanee. Non ci sono molte parole per esprimere la potenza di quello che questi uomini hanno percepito e, infatti, tutti usano, più o meno, le stesse parole.

Il fatto che da quella distanza puoi alzare il tuo pollice e puoi nascondere la Terra dietro quel pollice. Tutto ciò che hai conosciuto, ciò che ami, le tue preoccupazioni, i problemi del mondo intero dietro a un dito...

(Jim Lowel)

Più ci allontanavamo più [la Terra] diventava piccola, fino a quando non si è ridotta alle dimensioni di una biglia. Questo oggetto bellissimo, vivo, sembrava cosi fragile, cosi delicato da potersi sbriciolare solo toccandolo con un dito. Vedere questo non può che cambiare un uomo.

(James B. Irwin)

Queste parole non esprimono concetti, non argomentano, vengono direttamente dai sensi. Gli astronauti vedono finalmente il nostro pianeta da lontano, nella giusta prospettiva, nella sua rotondità. Lo per- 
cepiscono finalmente come un oggetto unitario, disperso in un universo maestoso, buio e inospitale Molti cercano di esprimere un moto di affetto profondo e costruttivo per quell'oggetto fragile e per chi lo abita, un'intuizione potente che sembra, per un attimo, spazzare via le nebbie e le trappole ideologiche del ventesimo secolo. Alcuni si spingono a sostenere che quella visione spettacolare, se fosse accessibile ai più (inclusi i potenti del mondo), potrebbe convincere l'intera comunità umana a ricominciare daccapo, a mente fresca. Un poeta americano, Archibald McLeish cercherà di interpretare lo stato d'animo degli astronauti in poche righe ecumeniche riportate sotto la prima fotografia della Terra vista per intero, pubblicata sulla prima pagina del "New York Times" il 25 Dicembre 1968:

Vedere la terra veramente com'è, piccola e azzurra e meravigliosa nel silenzio eterno in cui fluttua, significa vedere noi stessi come abitanti della Terra, tutti insieme, fratelli su quell'incanto luccicante in mezzo al gelo eterno: fratelli consapevoli di essere tali".

Leggendo questi resoconti si ha quasi l'impressione che il gesto di voltarsi a guardare la Terra dalla Luna sia riuscito, seppure per un attimo, là dove la cultura aveva fallito per millenni: l'uomo vede finalmente le cose come stanno e diviene consapevole della loro vera dimensione, del loro valore. Come se la rivoluzione copernicana si fosse incarnata in un gesto, manifestando il suo significato più profondo con più di quattrocento anni di ritardo. Certo, è difficile descrivere le vertigini che hanno provato gli astronauti nel momento esatto in cui hanno toccato, letteralmente con un dito, la meravigliosa modestia della Terra. In fondo, è anche inutile provarci; certi fatti sono dirompenti solo nella loro immediata concretezza, quando obbligano i nostri sensi a toccare il mistero delle cose per quello che sono. Nel 1968 si sapeva già che la Terra era rotonda, periferica e infinitamente piccola in un universo immenso; ma un conto è saperlo, un altro è sentirlo.

Come possiamo risvegliare i nostri sensi alla coscienza? Esiste un modo, un gesto sublime, per sentire in prima persona il mistero della coscienza per quello che è, in tutta la sua potenza? Forse esiste, anche se si tratta, a differenza dell'esperienza vissuta dagli astronauti, di qualcosa che non ha nulla di eroico, qualcosa che di solito si tende a non raccontare per una questione di buon gusto. Infatti, per avvicinarsi a questo gesto ci tocca dimenticare l'atmosfera asettica e maestosa dello spazio extraterrestre e infilarci nei sotterranei asfittici di una camera mortuaria, 
nei panni di un povero studente di medicina che lotta contro un potente senso di nausea; la prima autopsia è un'esperienza forte che non si dimentica. Non appena l'anatomopatologo seziona il torace e l'addome del cadavere adagiato sul tavolo di ferro, crollano gran parte delle sicurezze faticosamente acquisite in anni di studio: i confini tra gli organi non sono così distinti come lo erano nelle illustrazioni del testo di anatomia, il fegato non è così marrone, $\mathrm{i}$ polmoni non sono così blu e il cuore non è affatto così rosso. In un attimo, gli ingranaggi, le leve, le pompe, i filtri e tutti gli altri meccanismi cristallini della fisiologia si sciolgono in una poltiglia omogenea mentre l'effluvio della decomposizione penetra le mascherine inutilmente imbevute di dopobarba. E' una brutale rivelazione: siamo fatti di una materia poco elegante e facilmente deperibile. Anche lo studente di medicina più cinico, in fondo, resiste a concepire il proprio corpo come qualcosa di veramente materiale; la prima autopsia scardina d'acchito questa resistenza, ma questo è solo l'inizio.

Dopo aver esaminato gli organi interni di torace e addome, l'anatomopatologo incide lo scalpo e fa breccia nel cranio del cadavere con lo stridore di un seghetto circolare. Due o tre colpi di scalpello ben assestati e la calotta cranica, rimossa, viene appoggiata sul tavolo metallico con un rumore sordo. Sono suoni che lasciano il segno, che rimbombano nelle orecchie per giorni, come può riecheggiare il tonfo di un incidente stradale in cui si è rimasti coinvolti. Dopo aver liberato la convessità del cervello dalle membrane che lo avvolgono, l'esaminatore solleva il lobo frontale con una spatola e seziona con una forbicina i due nervi ottici, poi il nervo olfattivo, i nervi acustici e tutte le fibre che ancora legano l'encefalo alla scatola cranica; per ultimo, taglia il midollo e il cervello è libero. Ora, il medico appoggia l'organo, appena estratto, sulle mani aperte dello studente più vicino, il quale si appresta a esaminarlo. Il cervello passa, di mano in mano, da uno studente all'altro e presto arriva il vostro turno; immaginate che ora tocchi a voi. Potete scegliere di analizzare quest'organo, come avete appena fatto con milza, fegato e cuore, per poi passarlo al vostro collega. Oppure, potete fermarvi un istante e pensare che, solo fino a qualche ora prima, questo pezzo di materia umida e gelatinosa, che adesso pesa sul palmo della mano, ospitava un universo vasto come il vostro. Tutto ciò che siete, tutto ciò che conoscete, che ricordate, immaginate e sognate è contenuto in un oggetto che si maneggia come un qualsiasi altro oggetto di questo mondo. Un pezzo di materia che ha dei confini e un peso. Adesso, avete le vertigini; vertigini più forti di quelle che avete provato quando 
da bambini, prima di addormentarvi, pensavate, con tutta l'intensità di cui eravate capaci, all'infinità del cielo e al numero delle stelle. Forse, più forti di quelle che hanno ubriacato gli astronauti nell'istante in cui hanno visto tramontare il nostro piccolo pianeta dietro la luna. Fermarsi a soppesare il cervello in una mano è un'esperienza dirompente che dissolve, in un istante, il peso delle consuetudini, delle posizioni filosofiche, delle definizioni e delle trappole logiche che ci allontanano dal mistero della coscienza. Una domanda, urgente e semplice, sembra venire direttamente dai sensi, dai nervi della mano che soppesa: che cos'ha di diverso, di speciale, questo oggetto?

In piedi, di fronte al tavolo anatomico, non vi interrogate su come la materia del cervello possa ospitare le contorsioni dell'autocoscienza e neppure sentite il bisogno di capire come il cervello riesca a produrre la percezione di una scena complicata simile a quelle raffigurate nei un quadri di Brueghel; avete appena esaminato il fegato, il cuore e vi chiedete come possa quest'organo di un chilo e mezzo contenere un soggetto che, semplicemente, vede luce o. Perché il cervello sì e gli altri organi no? Da bravi studenti, vi ricordate che il cervello genera segnali elettrici, ma subito vi viene in mente che questo lo può fare anche il cuore. Per un attimo vi illuminate e pensate che il cervello è composto da decine di miliardi di neuroni e da una quantità incalcolabile di sinapsi; poi, però, vi sovviene che il cervelletto, un altro pezzo di materia contenuto nel cranio che non ha a che fare con la coscienza, possiede ancora più neuroni del cervello. Guardate il cervelletto adagiato in una bacinella sul tavolo anatomico e vi chiedete come ciò sia possibile, ma subito vi rapisce un altro pensiero: durante il corso di fisiologia, avete imparato che, in certe fasi del sonno, il cervello si isola temporaneamente dai nervi che lo collegano al mondo esterno per generare, da solo, la movimentata fiera di un sogno; universi vividi, chiassosi e colorati affollano il cranio nel silenzio e nel buio di ogni notte. Vi sconvolge l'idea che quel fragile tessuto, se fosse ancora perfuso da una soluzione di ossigeno e zuccheri, potrebbe sognare, lì sul palmo della vostra mano. Così, mentre annaspate, qualcuno vi dice che il tempo a vostra disposizione è scaduto; cedete la massa molliccia nelle mani del compagno al vostro fianco e le vertigini vi abbandonano. Il tutto è durato poco più di un minuto, ma mai un mistero fu così intenso e tangibile.

Chi scrive ha vissuto, quando era studente di medicina, un'esperienza di questo genere ed è convinto che soffermarsi a sentire il peso del cervello con una mano sia, più o meno, come nascondere la Terra 
dietro un dito a 300 mila chilometri di distanza: un'esperienza sublime - nell'accezione filosofica e letteraria del termine - sicuramente angosciante e potenzialmente liberatoria. All'inizio, ci turba accostare la perfezione, la bellezza e l'assolutezza di tutto ciò che possiamo concepire e creare quando ci troviamo in uno stato di grazia a un oggetto così umile: una piccola macchina oleosa i cui ingranaggi, prima o poi, si incepperanno e si fermeranno. Tutti i significati sembrano perduti e ci assale una profonda angoscia, un moto d'istintiva repulsione: non può essere così, noi siamo molto più di questo! Poi, incrociamo lo sguardo vuoto del cadavere e non possiamo fare a meno di pensare che anche lui era un essere speciale, infinito, uno come noi. Gli astronauti hanno visto la straordinaria varietà del mondo in una piccola biglia sperduta in uno spazio immenso; e noi abbiamo toccato la modesta materia che conteneva la straordinaria ricchezza di un uomo cosciente. Come gli astronauti, non possiamo più far finta di nulla: le cose vanno accettate per quello che sono. Intuiamo che se vogliamo dare un significato a tutto ciò è necessario capire il cervello, l'unico pianeta che veramente abitiamo, per apprezzarlo e rendergli giustizia. L'istinto a capire è ormai troppo forte, irresistibile. Durante l'autopsia, per un attimo, abbiamo avuto la certezza che l'oggetto fisico che stavamo per passare al nostro compagno di studi avesse qualcosa veramente speciale, di sostanzialmente unico. Dobbiamo capire cosa!

Il problema si pone quando cerchiamo di sezionare il problema con le lame della logica. Se una mattina ci svegliamo e ci ricordiamo di un sogno particolarmente interessante, ci piace richiamarlo, ripensarlo e manipolarlo nella nostra mente. Tuttavia, i sogni sono impressi su una pellicola molto labile e basta toccarli con il pensiero che perdono di colore e intensità. La stessa cosa accade con il mistero della coscienza; a volte, lo possiamo sentire in tutta la sua potenza, poi però, se ci pensiamo troppo, il mistero sbiadisce e l'istinto a svelarlo si indebolisce. Questo accade non di rado. Per esempio, alcuni filosofi [3] hanno concepito la possibilità logica che esistano degli "zombies", ovvero, creature che sono, dal punto di vista fisico, identiche a noi che, tuttavia, agiscono in modo del tutto automatico, meccanico, non consapevole e privo di coscienza. Questi zombies sono diversi da quelli evocati dagli stregoni caraibici e diversi dai corpi senza vita parzialmente decomposti che popolano i film dell'orrore; gli zombi evocati dai filosofi sono creature educate, rispettabili e assolutamente indistinguibili da noi dal punto di vista fisico. Nessun esame medico, nessun test psicologico, 
nessun osservatore noterà mai alcuna differenza tra un essere umano e uno zombi filosofico. L'unica differenza è che gli zombi filosofici sono completamente privi di coscienza. Se toccano una superficie rovente, gli zombi ritraggono l'arto, urlano e imprecano come noi, ma non provano alcun dolore. Infatti, non si prova assolutamente nulla a essere uno zombi. Secondo molti filosofi contemporanei l'esistenza di una tale creatura, o meglio, la possibilità della loro esistenza (il semplice fatto che voi abbiate capito quello che avete appena letto) dimostrerebbe che la coscienza non è riducibile ai processi cerebrali. In altre parole, il solo fatto che sia possibile concepire una creatura che dal punto di vista materiale è fatta come noi ma che non ha alcuna esperienza soggettiva (Fig. 1A) sarebbe la dimostrazione che non vi è alcuna necessità logica di derivare la coscienza da una descrizione, anche completa, dello stato fisico di un altro essere umano.

Un altro esperimento logico [4] viene impiegato per supportare una simile conclusione: Mary è una scienziata esperta dei processi cerebrali che sottendono la visione a colori. Mary sa tutto quello che c'è da sapere sui meccanismi fisici, biologici del cervello coinvolti nella visione a colori: conosce come il cervello sia in grado di discriminare tra lunghezze d'onda diverse lungo lo spettro della luce e di come sia in grado di elaborare gli stimoli visivi integrando le varie informazioni. Conosce tutti i nomi dei colori. Tuttavia, Mary, ha sempre vissuto in un ambiente dove può vedere solo il bianco, il nero e le tonalità di grigio. E, infatti, c'è solo una cosa della quale Mary non è ancora a conoscenza: della sensazione di vedere un colore. Questo "argomento della conoscenza" viene spesso utilizzato, così come l'argomento dello "zombie filosofico", per sostenere l'idea che l'esperienza cosciente non può essere ridotta agli stati fisici del cervello, né tantomeno può essere spiegata. Queste (e molte altre) argomentazioni, sono logicamente sostenibili, suscitano dibattiti accesi e interessanti ma, alla lunga, rischiano di appesantire ed offuscare il nostro istinto a capire. Durante l'autopsia, non abbiamo potuto fare a meno di sentire che l'oggetto fisico che tenevamo in mano aveva qualcosa di speciale, qualcosa che lo rende diverso dagli altri oggetti fisici. Allo stesso modo, non possiamo fare a meno di cercare i segni della coscienza nella materia quando ci troviamo di fronte ad un paziente che, uscito dal coma, giace immobile e muto in un reparto di terapia intensiva. Questa, però, non è più una questione esistenziale, è una questione etica. 


\section{UNIVERSI NASCOSTI}

Nelle settimane che seguono un grave insulto cerebrale (di origine traumatica, vascolare, o anossica) capita che il paziente entri in coma. Il coma è uno stato transitorio, che dura poche settimane, durante il quale il soggetto giace, ad occhi chiusi, in uno stato simile al sonno; un sonno da cui, tuttavia, non può essere risvegliato. Nel giro di qualche settimana, tipicamente, i pazienti in coma, o precipitano verso un quadro di morte cerebrale, o si svegliano e aprono gli occhi. Quando questo ritorno della vigilanza è solamente associato a movimenti riflessi, in assenza di qualsiasi segno di interazione volontaria con l'ambiente, il paziente viene clinicamente classificato come "vegetativo" [5]. Lo stato vegetativo è una condizione in cui si presume che la coscienza sia completamente abolita. Lo stato vegetativo può rappresentare uno stato di transizione verso un ulteriore recupero, o può persistere per anni. Segni, anche labili, di attività motoria volontaria caratterizzano l'evoluzione verso lo stato di minima coscienza. Questa condizione, di recente definizione [6] [7] identifica quei pazienti che sono emersi dallo stato vegetativo ma che sono ancora incapaci di comunicare i propri pensieri e le proprie emozioni. L'uscita dallo stato di minima coscienza è segnato dal recupero della capacità di instaurare una comunicazione verbale, o non verbale, funzionale. In rari casi, alcuni pazienti emergono dal coma direttamente in uno stato di piena coscienza ma rimangono completamente paralizzati, eccetto che per i movimenti oculari verticali [8].

In pratica, la distinzione clinica tra stato vegetativo (assenza di coscienza) e le altre condizioni, in cui è presente un livello variabile di esperienza cosciente, è tutt'altro che facile. Questo perché, normalmente, valutiamo la presenza di coscienza nei nostri simili in un modo che, a pensarci bene, è piuttosto superficiale. Essenzialmente, nella vita quotidiana, e nella pratica clinica, misuriamo il livello di coscienza di altri soggetti basandoci sulla loro capacità di interagire e comunicare con l'ambiente circostante. Facciamo questo anche se sappiamo bene che la coscienza può essere interamente generata all'interno del cervello, in assenza di qualsiasi interazione con il modo esterno; ciò accade, quasi ogni notte, quando sogniamo (durante la fase REM del sonno siamo disconnessi dall'ambiente esterno, siamo paralizzati, ma siamo coscienti). A causa di questa discrepanza, non di rado, può accadere che la presenza di coscienza venga misconosciuta, o sottovalutata, in pazienti portatori di gravi lesioni cerebrali, i quali sono pienamente coscienti, ma 
completamente paralizzati. Un articolo recentemente pubblicato sulla rivista scientifica Science [9] ha portato questo problema all'attenzione dei media e dell'opinione pubblica. L'articolo riporta il caso di una giovane donna che, a seguito di un evento traumatico cerebrale, era stata dichiarata in stato vegetativo persistente. Qualche mese dopo, la paziente era stata sottoposta ad una registrazione dell'attività cerebrale in risonanza magnetica funzionale (f-MRI), mentre le venivano somministrate delle istruzioni verbali ("immagina di giocare a tennis", oppure, "immagina di camminare nel tuo appartamento"). Per quanto incapace di comunicare con l'esterno e di compiere qualsiasi atto motorio finalizzato, la paziente risultava sorprendentemente in grado di rispondere alle istruzioni producendo volontariamente schemi di attività cerebrale specifici. In altre parole, il cervello di questa donna aveva conservato, profondamente nascosta al suo interno, una significativa capacità di comprendere e di produrre attività volontaria che era stata misconosciuta, nonostante mesi di attenta valutazione clinica.

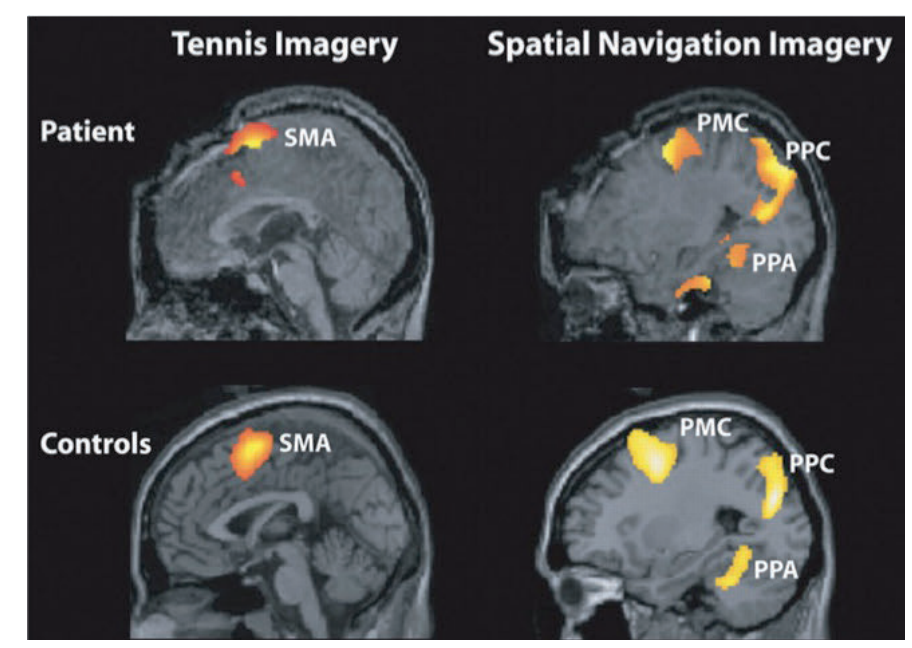

Fig. 1. Attivazioni neuronali rilevate tramite risonanza magnetica funzionale in soggetti che immaginano di giocare a tennis (figure a sinistra) o di navigare nel proprio appartamento (figure a destra). Le immagini nel pannello superiore si riferiscono alla giovane paziente la cui storia è riportata nel testo. Un'estesa lesione cerebrale è chiaramente visibile in corrispondenza della corteccia frontale. La paziente era in grado di produrre attivazioni neuronali specifiche per ciascun comando (tennis e navigazione) che erano indistinguibili da quelle precedentemente osservate in soggetti sani di controllo (pannello inferiore). Da Owen et al., "Detecting awareness in the vegetative state", Science 2006. 
Questo test nello scanner f-MRI rivela la nostra incapacità di vedere la coscienza quando questa non si manifesta apertamente tramite gesti e parole [10] ma non risolve il problema di fondo. Infatti, esistono molti pazienti, che per quanto coscienti, non sono in grado di dare segni di sé anche quando esaminati nello scanner f-MRI. In particolare, pazienti cerebrolesi affetti da afasia, mutismo acinetico, depressione catatonica o ancora diffuse lesioni dopaminergiche possono non capire le istruzioni dell'esaminatore, non aver la motivazione, le risorse attentive o cognitive per immaginare di giocare a tennis e per portare a termine con successo questo compito. Di fatto, una percentuale elevatissima $(90 \%)$ di pazienti che si trovano in uno stato clinico di minima coscienza falliscono questo test [11] .

Questi casi drammatici (tuttavia, non rari) situati ai confini della neurologia clinica sollevano un problema di carattere generale: sappiamo bene che la coscienza non dipende dalla capacità di interagire con l'ambiente esterno e di comunicare e sappiamo che l'esperienza cosciente è interamente generata all'interno del cervello; tuttavia, non abbiamo modo di estrarre direttamente dal cervello un indice oggettivo della presenza di coscienza. In pratica, questo problema si traduce in un'elevata incidenza (fino al $43 \%$ ) di diagnosi errate: molti, troppi pazienti che sono in realtà coscienti vengono erroneamente etichettati come incoscienti [12]. Questo dato mette in luce un problema etico generale, che va ben oltre la sfera della pratica clinica: se non svilupperemo un metodo affidabile per misurare la coscienza ci potremmo trovare nella situazione paradossale di non riconoscere la coscienza dove essa è presente e di vederla dove essa non c'è. Infatti, non solo stiamo salvando pazienti complessi in cui sopravvivono ampie isole di tessuto cerebrale largamente disconnesse dall'ambiente esterno, ma stiamo anche producendo cervelli artificiali le cui performance e capacità di interazione migliorano, e continueranno a migliorare, con un rateo esponenziale. E' più cosciente il paziente che giace immobile come una pietra o il supercomputer che vince a scacchi e che risponde a qualsiasi domanda? Se non noi, sicuramente i nostri figli si troveranno in difficoltà, se continueremo a misurare la coscienza sulla base del solo comportamento.

\section{UNA SFIDA DIFFICILE}

I problemi posti dai pazienti (e quelli che porranno i prossimi sviluppi dell'intelligenza artificiale) sottolineano la necessità di identifica- 
re, e misurare, i meccanismi fondamentali che rendono un sistema fisico capace di coscienza. Forse è bene assecondare la curiosità istintiva del povero studente di medicina che improvvisamente si ritrova con un cervello sul palmo della mano e mettere da parte lo scetticismo del filosofo; forse, vale veramente la pena di imbarcarsi in un'avventura alla ricerca delle proprietà di base che rendono un particolare chilo e mezzo di materia capace di immaginare e di sognare. Conviene cominciare questo viaggio dagli ostacoli più grandi, ossia da tre paradossi (irrisolti) del rapporto cervello-coscienza, quelli che giustificano un certo scetticismo anche nello studioso più propositivo e ottimista. Il primo paradosso riguarda il confronto tra due particolari pezzi di materia, la corteccia cerebrale ed il cervelletto. Sappiamo da tempo che la corteccia cerebrale, che riveste gli emisferi del cervello, è essenziale per la coscienza: lesioni diffuse della corteccia cerebrale e dei nuclei talamici sottostanti portano invariabilmente alla scomparsa, temporanea, o irreversibile, della coscienza. Il cervelletto è un altro pezzo di cervello situato nella fossa cranica posteriore. Quando, per un trauma o per un tumore, si asporta l'intero cervelletto, la coscienza non è affatto alterata. Nonostante un paziente senza cervelletto abbia gravi problemi di coordinazione motoria, il flusso della sua esperienza cosciente continua come prima, con la stessa ricchezza, e con la stessa intensità. Chiaramente, è cruciale capire le ragioni di questa paradossale asimmetria tra il contributo dato alla coscienza da due strutture nervose apparentemente altrettanto complicate. Il cervelletto ha infatti persino più neuroni della corteccia cerebrale (80 miliardi contro 30 miliardi), dispone di una rete di comunicazioni altrettanto abbondante e sofisticata, contiene altrettante sostanze chimiche, ed ha intensi rapporti con il mondo esterno: importa segnali visivi, acustici, tattili e vari altri, ed esporta comandi motori che sono in grado di regolare molti aspetti del comportamento. Perché quindi la corteccia cerebrale genera la coscien$\mathrm{za}$, ma il cervelletto no?

Un secondo problema consiste nel capire cosa cambia nel cervello quando ci addormentiamo (o quando veniamo anestetizzati) e la coscienza svanisce. Perché a volte la materia del cervello genera un universo di coscienza e altre volte no? Cosa fa la differenza? Sappiamo che la scatola cranica è affollata da miliardi di neuroni e sappiamo che ciascuno di questi neuroni può emettere, ogni secondo, una quantità variabile di segnali elettrici (da zero a circa 400, ogni secondo), detti potenziali d'azione. Fino a qualche decennio fa si pen- 
sava che la coscienza svanisse durante sonno perché il cervello si "spegneva”. Ora, però, sappiamo che le cose non stanno così: registrazioni dell'attività elettrica e del metabolismo [13] ci dicono che i neuroni sono tutto fuorché spenti durante sonno. Anche durante le fasi più profonde del sonno NREM, quando la coscienza si restringe fino a sparire, è possibile osservare livelli di attività neuronale più alti di quelli tipici della veglia [14]. Qualcosa di molto simile durante le crisi epilettiche generalizzate [15], quando la coscienza abbandona un cervello che è addirittura iperattivo. Recentemente, grande attenzione è stata dedicata al fenomeno della sincronizzazione del ritmo "gamma", ossia alla capacità di diversi gruppi neuronali emettere in modo coordinato segnali ad una frequenza piuttosto elevata (circa 40 impulsi al secondo). In particolare, l'idea che ci sia un legame causale tra la sincronizzazione "gamma" e la coscienza deriva dall'osservazione sperimentale che la percezione cosciente di un oggetto complesso, come un volto, è accompagnata dalla produzione di onde rapide di attività elettrica che sono sincrone in diverse aree del cervello. Questa interessante osservazione ci dice che per percepire un oggetto complesso è importante che i neuroni si coordinino tra di loro. Tuttavia, anche la sincronizzazione, così come il livello di attività neuronale, non correla in tutto e per tutto con la presenza di coscienza; infatti, ritmi di scarica neuronale veloci ed iper-sincroni possono essere ancora presenti durante anestesia [16], durante crisi epilettiche generalizzate e in stato di coma [17]. Di fatto, sfortunatamente, la misura di queste due proprietà fisiche (livelli di attività e sincronizzazione) non ci aiutano a decidere se un paziente che giace muto ed immobile in un letto di terapia intensiva è cosciente, o meno.

Un terzo paradosso è il sogno. Mentre, come abbiamo appena visto, durante le fasi profonde del sonno NREM della prima parte della notte la coscienza abbandona il cervello, più tardi durante il sonno della mattina, e in particolare durante il sonno REM, questa ritorna più vivida e cangiante che mai, nella forma di un sogno [18]. Nel sogno, il cervello, completamente disconnesso dal mondo esterno, diventa regista e produce un intero universo fatto di luoghi, animali, persone, colori e di cose mai viste prima. La coscienza onirica è completamente invisibile all'osservatore esterno, la può solo rivelare il soggetto al risveglio, ammesso che se ne ricordi. In principio, l'esperienza di un sogno potrebbe essere prodotta da un cervello senza un corpo, isolato in una bacinella e perfuso da un'appropriata soluzione di ossigeno e nutrienti. 
Più realisticamente, è possibile che molti soggetti con gravi lesioni dei sistemi motori e sensoriali si trovino in uno stato onirico, isolati e coscienti di un mondo a noi del tutto inaccessibile. Fondamentalmente, il sogno ci insegna che la coscienza può esistere indipendentemente dai sensi e dalla capacità di agire e in assenza un mondo esterno. Chiaramente, le proprietà fisiche fondamentali della materia cosciente, quelle di cui siamo alla ricerca, devono essere presenti e misurabili in un cervello che sogna.

I fatti riguardanti il rapporto cervello-coscienza appena descritti sono tanto reali quanto paradossali. Chiaramente, se non risolviamo questi paradossi fondamentali, non saremo mai in grado di dire alcunché di significativo sul rapporto materia-coscienza; tantomeno, saremo capaci di dire se un paziente che si sveglia dal coma muto e immobile è cosciente. Se le osservazioni empiriche ci stimolano e ci confondono allo stesso tempo, da dove partiamo? Che cosa dobbiamo cercare nella materia del cervello? Per orientarci in questo panorama complicato possiamo fare un passo indietro e guadagnare un po' di prospettiva. Questo passo lo possiamo fare grazie a una teoria della coscienza, la teoria dell'informazione integrata (IIT), recentemente proposta da Giulio Tononi [19] [20]. La IIT si pone proprio l'obbiettivo di formulare une legge generale, che permetta di dare coerenza alle osservazioni sperimentali accumulate, di formulare predizioni specifiche e di proporre una misura oggettiva della coscienza. Dopotutto, l'istinto che seguiamo è quello di pesare, di misurare anche grossolanamente, ciò che conta perché la materia generi coscienza.

\section{LA LUCE DI UNA TEORIA}

Secondo la teoria dell'informazione integrata, un sistema fisico è cosciente nella misura in cui è in grado di integrare informazione. Questa definizione apparentemente piuttosto criptica e astratta prende origine proprio dalla fenomenologia della coscienza, ovvero dall'osservazione delle due caratteristiche fondamentali dell'esperienza soggettiva: 1) l'esperienza cosciente è ricchissima di informazione nel senso che è differenziata, ossia il repertorio potenziale di diversi stati di coscienza è straordinariamente grande 2) l'esperienza cosciente è integrata, ossia ogni stato di coscienza è esperito come una singola entità. Ricchezza di informazio- 
ne e integrazione sono due caratteristiche talmente necessarie e talmente connaturate al fluire della nostra esperienza quotidiana che fatichiamo a riconoscerle. Per metterne in evidenza l'importanza è possibile ricorrere a due esperimenti immaginari che riportiamo di seguito.

Informazione. Immaginiamo di trovarci di fronte ad uno schermo omogeneo che cambia ogni pochi secondi da acceso a spento, e di doverne comunicare lo stato dicendo "chiaro" o "scuro". Di fronte allo schermo c'è anche un fotodiodo, un semplicissimo congegno elettronico che sa indicare la presenza o assenza di luce. Il primo problema della coscienza si riduce a questo: quando distinguiamo tra schermo acceso e spento, ciascuno di noi ha l'esperienza soggettiva di chiaro e di scuro: "vediamo" uno schermo chiaro o scuro. Il fotodiodo sa distinguere altrettanto bene tra chiaro e scuro, ma presumibilmente non ha alcuna esperienza soggettiva. Qual è la differenza fondamentale che rende noi coscienti ed il fotodiodo no?

Secondo la teoria, la differenza fondamentale è la seguente: quando il fotodiodo indica "chiaro", distingue solamente tra due stati; quando noi diciamo "chiaro", distinguiamo in realtà non solo tra chiaro e scuro, ma tra chiaro e miliardi di miliardi di alternative. Per esempio, lo schermo potrebbe inaspettatamente mostrare, anziché una superficie omogenea chiara o scura, un qualunque fotogramma tratto da un qualunque film. Ci sono milioni di fotogrammi e milioni di film eppure, senza alcuno sforzo, e nel giro di una frazione di secondo, ciascuno di essi indurrà in noi un'esperienza cosciente diversa. Il fotodiodo invece non può far altro che continuare ad indicare uno o l'altro di due stati. Questo esperimento immaginario suggerisce che la differenza fondamentale tra noi ed il fotodiodo sta nella quantità di informazione a disposizione. L'informazione, classicamente [21], è una misura di quanto è grande il repertorio di alternative: quanto maggiore è il numero di alternative, tanto maggiore sarà il numero di bit di informazione prodotta. Per noi, la visione di uno schermo chiaro esclude un numero straordinariamente grande di alternative, ed è pertanto straordinariamente informativa (anche se tipicamente non ci facciamo caso). Per il fotodiodo, invece, uno schermo chiaro esclude soltanto uno schermo scuro, e l'informazione è pari ad un solo bit. La ricchezza di informazione, sostiene la teoria, è una proprietà fondamentale dell'esperienza cosciente. E' infatti così fondamentale che la diamo tutti per scontata. 
Integrazione. L'informazione, tuttavia, non basta a spiegare la coscienza se non va di pari passo con l'integrazione. Consideriamo, per esempio, una macchina fotografica digitale idealizzata, con un sensore costituito da un milione di fotodiodi. Sembrerebbe che, con un gran numero di fotodiodi, sia possibile distinguere tra un' enorme numero di alternative: basta puntare l'obbiettivo in direzioni diverse, ed il sensore è in grado di "rispondere" in modo diverso a miliardi di scene diverse. L'informazione a disposizione del milione di fotodiodi del sensore è enormemente maggiore di quella a disposizione di un singolo fotodiodo, eppure nessuno penserebbe che la macchina "veda" coscientemente. Qual è, allora, la differenza fondamentale tra noi e la macchina fotografica?

La risposta è che il sensore della macchina fotografica non è che una collezione di un milione di fotodiodi indipendenti, ciascuno in grado di distinguere tra chiaro e scuro, e non un sistema integrato capace di distinguere tra miliardi di immagini. Questo perché tra i fotodiodi non è possibile alcuna interazione causale - alcuno scambio di informazione. E infatti, se il sensore venisse tagliato longitudinalmente in due parti (o in un milione di parti), il funzionamento della macchina fotografica non cambierebbe. Non è così nel nostro caso: se le cellule del nostro cervello venissero mantenute in grado di funzionare, ma fossero disconnesse le une dalle altre, non c'è dubbio che la coscienza scomparirebbe. Basta pensare a cosa accade quando si divide il cervello in due parti tagliando il corpo calloso. Come dimostrato dai famosi studi condotti dal premio Nobel Roger Sperry [22], ciò che accade è che si divide in due anche la coscienza, col risultato che due coscienze indipendenti finiscono per condividere lo stesso cranio.

In sintesi, la teoria proposta da Tononi prende spunto dalla fenomenologia e suggerisce che la capacità di un sistema fisico di generare coscienza dipende da un bilancio ottimale tra informazione e integrazione, ovvero, da un delicato ed improbabile equilibrio tra diversità e unità. La teoria propone anche una misura dell'informazione integrata, chiamata phi (, dove la barra verticale della lettera greca indica informazione e il cerchio l'integrazione). Per misurare occorre stimolare in tutti i modi possibili i vari elementi che costituiscono il sistema e osservare le risposte che vengono generate tramite le interazioni tra questi elementi. In questo modo misura la quantità di stati che un sistema fisico è in grado di discriminare come un tutt'uno. 
Simulazioni al calcolatore, effettuate su sistemi estremamente semplici hanno dimostrato che l'integrazione dell'informazione è ottimizzata (valore di elevato) quando gli elementi di un insieme sono connessi in modo tale da essere allo stesso tempo funzionalmente specializzati (i pattern di connessione sono differenti per elementi differenti) e funzionalmente integrati (tutti gli elementi possono essere raggiunti da tutti gli altri elementi del network). Se la specializzazione funzionale è persa sostituendo una connettività eterogenea con una omogenea, oppure se l'integrazione funzionale è persa perché le connessioni formano piccoli moduli indipendenti, il valore di diminuisce considerevolmente.

Secondo la IIT, questo è esattamente il motivo per cui, tra le varie strutture del cervello (e non solo), il sistema talamo-corticale è così speciale: è naturalmente organizzato in un modo da enfatizzare contemporaneamente sia la specializzazione funzionale sia l'integrazione funzionale. Infatti il sistema talamo-corticale, comprende un vasto numero di elementi che sono funzionalmente specializzati, attivandosi in differenti circostanze [23]. Questo è vero a molteplici livelli, dai differenti sistemi corticali che si occupano della vista, dell'udito, alle differenti aree corticali che si occupano di forma, colore, movimento, ai differenti gruppi di neuroni che rispondono a differenti direzioni di moto. Allo stesso tempo, gli elementi specializzati del sistema talamo-corticale sono integrati attraverso un esteso network di connessioni intracorticali che permette una rapida ed efficace interazione tra le aree [24]. Il concetto di informazione integrata spiega anche perché il cervelletto non ha alcun peso per la coscienza: il cervelletto è costituito da una miriade di moduli specializzati (con diverse competenze) che sono tuttavia segregati. Il cervelletto è chiaramente modulare, non possiede connessioni a lunga distanza al suo interno e neppure un corpo calloso (il voluminoso fascio di fibre che connette i due emisferi cerebrali). Dal punto di vista dell'informazione integrata (e della coscienza), il cervelletto ha un peso simile a quello di una telecamera. In questa prospettiva il primo paradosso del rapporto cervello-coscienza risulta molto più comprensibile. A questo punto, che cosa possiamo dire riguardo ai paradossi del sonno, del sogno e dell'anestesia? A questo riguardo, la teoria predice esplicitamente che il dissolversi della coscienza nel sonno e nell'anestesia dovrebbe essere associato ad una riduzione dell'integrazione all'interno del sistema talamo-corticale (che si 
disgregherebbe in moduli causalmente indipendenti), o ad una riduzione dell'informazione (il restringimento del repertorio possibile ad un piccolo numero di stati stereotipati), o a entrambe. Questa specifica predizione è però difficile da testare nell'uomo, dal momento che, in pratica, a tutt'oggi può essere misurata rigorosamente solo per piccoli sistemi simulati. E' però possibile, almeno in principio, approssimare la misura teorica per ottenere una valutazione grossolana della capacità del cervello di integrare informazione. Tanto basta per verificare empiricamente alcune predizioni di base, per affrontare alcuni paradossi irrisolti e, magari, per svelare un barlume di coscienza nel buio di un reparto di terapia intensiva.

\section{UN'APPROSSIMAZIONE EMPIRICA}

Alla luce della teoria, per apprezzare in modo affidabile la capacità del cervello di generare coscienza (definita come informazione integrata), non basta misurare quanto i neuroni sono spontaneamente attivi e neppure basta misurare la sincronizzazione tra gruppi distanti di neuroni. Infatti, è difficile dire se degli elementi costituiscono davvero un sistema integrato o meno semplicemente osservando se la loro attività è in qualche modo coordinata: tutti gli orologi del mondo, per quanto ben sincronizzati, non costituiscono certo un sistema unitario. Di fatto, l'abilità di integrare informazione può essere dimostrata con certezza solo da un punto di vista causale. Per fare ciò, è necessario utilizzare un approccio di tipo perturbazionale ed esaminare in che misura i diversi elementi che compongono un sistema sono in grado di interagire causalmente come un tutt'uno (integrazione) per produrre risposte che sono complesse e differenziate (informazione). Tra l'altro, questa indagine dovrebbe essere condotta stimolando direttamente la corteccia cerebrale per evitare possibili filtri o blocchi sottocorticali. Infine, poiché le interazioni causali tra neuroni talamocorticali si sviluppano su una scala temporale di frazioni di secondo, (proprio come la coscienza fenomenica), è molto importante registrare gli effetti neurali della perturbazione con l'appropriata risoluzione temporale. In sostanza, si dovrebbe trovare un modo di stimolare un sottoinsieme di neuroni corticali e misurare, con buona risoluzione spazio-temporale, gli effetti prodotti da questa perturbazione locale nel resto del sistema talamocorticale (Fig. 2). 


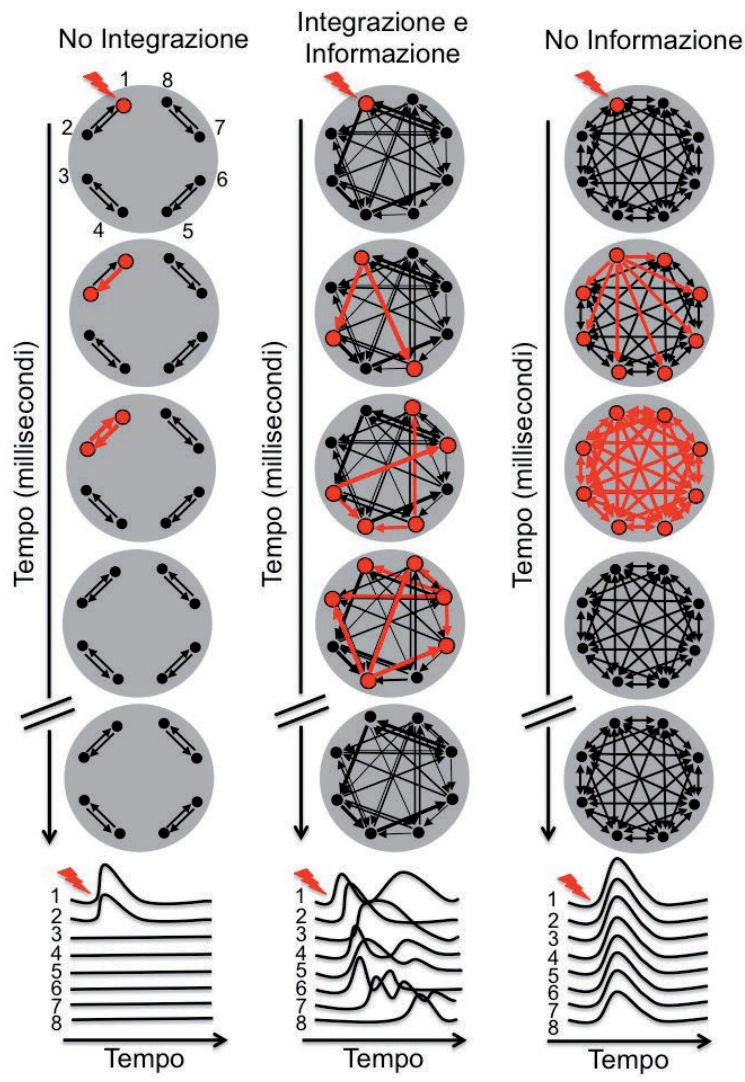

Fig. 2. Un'approssimazione empirica della misura teorica. In questa figura si utilizzano degli schemi semplificati allo scopo di illustrare il razionale della misura empirica dell'informazione integrata. Nel sistema modulare di sinistra (no integrazione), la perturbazione dell'elemento 1 è seguita da un'attivazione locale che coinvolge soltanto l'elemento 2 e che si esaurisce in breve tempo. Nel sistema a connettività omogenea raffigurato a destra (no informazione), l'attivazione dell'elemento 1 coinvolge immediatamente tutti gli altri elementi producendo così una risposta che è sì globale, ma semplice e stereotipata. In questo caso, a seguito della stimolazione dell'elemento 1, tutto si accende e poi tutto si spegne all'unisono, rivelando una mancanza di differenziazione all'interno del sistema (tutto è connesso con tutto, in egual modo). Il sistema al centro, caratterizzato dalla coesistenza di integrazione e differenziazione, reagisce alla stimolazione iniziale con uno schema di attività molto complesso. Solo in questo caso la risposta è allo stesso tempo, globale e differenziata: l'attività innescata nel sistema dalla perturbazione coinvolge elementi diversi, posti a distanza tra loro, a tempi diversi, dando luogo a uno schema di attività spazio-temporale complesso e durevole. Le tracce sotto i rispettivi schemini riportano l'andamento temporale delle attivazioni innescate dalla perturbazione nei diversi elementi. 
Oggi, questa misurazione può essere effettuata in modo non invasivo nell'uomo, grazie allo sviluppo di una nuova tecnica elettrofisiologica, basata sulla combinazione di stimolazione magnetica transcranica (TMS) ed elettroencefalografia ad alta densità (hd-EEG) [25] (Fig. 3). Questa metodica rende finalmente possibile la misura diretta della interazioni causali all'interno della corteccia cerebrale [26] [27]. Mediante la TMS, è infatti possibile stimolare direttamente la corteccia cerebrale tramite un breve e forte impulso magnetico $(<1 \mathrm{~ms}, 2 \mathrm{~T})$ indotto da un solenoide di rame applicato alla superficie dello scalpo. Il rapido cambiamento nella forza del campo magnetico induce un flusso di corrente nel tessuto cerebrale, che risulta nell'attivazione della sottostante popolazione neuronale. La raffica sincrona di potenziali d'azione così ottenuta si propaga lungo le vie di connessione disponibili, producendo attivazioni nelle regioni corticali bersaglio. Integrando la TMS con sistemi di neuro-navigazione ad infrarossi basati sulla ricostruzione digitale del cervello del soggetto è anche possibile rendere la perturbazione controllabile e riproducibile. Infine, usando amplificatori EEG a canali multipli, compatibili con la TMS, è possibile registrare, a partire da pochi millisecondi dopo la stimolazione magnetica, l'impatto che la perturbazione, applicata localmente, ha nel resto del sistema.

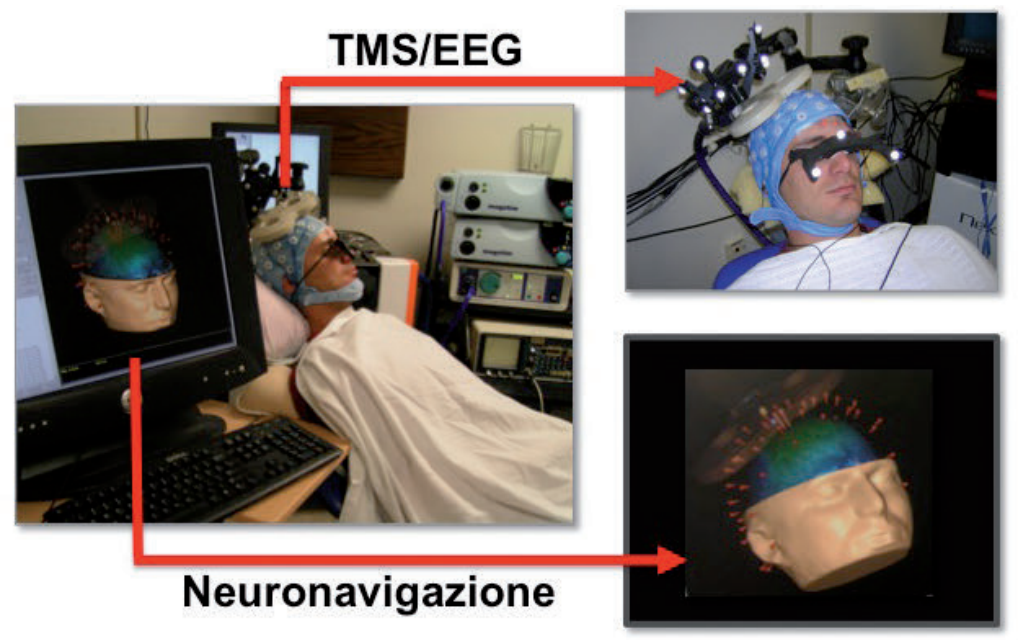

Fig. 3. Il set-up sperimentale utilizzato per le misure TMS-EEG. Lo stimolatore è posizionato su una porzione della corteccia frontale di sinistra, mentre il soggetto giace su una sedia reclinabile. Un sistema di neuro-navigazione guidato da immagini di risonanza strutturale consente il posizionamento preciso dello stimolatore. 
Dunque, almeno in linea di principio, la TMS/hd-EEG potrebbe rappresentare uno strumento appropriato per approssimare la misura teorica di informazione integrata nel cervello di un uomo. Se la coscienza ha a che fare con la capacità del cervello di integrare informazione, si dovrebbe dimostrare che le attivazioni evocate dalla TMS sono diffuse (integrazione) e complesse (informazione) in un cervello cosciente. A maggior ragione, si dovrebbe dimostrare che queste attivazioni divengono locali (rivelando una perdita di integrazione) e/o semplici (rivelando una perdita di informazione) quando la coscienza abbandona il cervello durante il sonno NREM e l'anestesia. Poi, dovremmo osservare un recupero dell'estensione e della complessità della risposta alla TMS quando la coscienza risorge in un cervello che, seppur disconnesso dall'ambiente sogna. Nella prossima sezione verranno sinteticamente descritti i risultati di misurazioni effettuate mediante TMS/hd-EEG durante sonno, sogno e anestesia.

\section{IL CERVELLO NEL SONNO, NEL SOGNO E NELL'ANESTESIA.}

La stimolazione diretta di un'area corticale applicata in un soggetto sveglio e cosciente risulta tipicamente in un pattern di risposta neuronale diffuso e complesso [28]. Come mostrato nella Fig. 4, durante lo stato di veglia, la TMS innesca un pattern di attività dove aree corticali diverse e lontane tra loro si attivano in tempi diversi (fino a 300 millisecondi). Questo schema di attivazione è precisamente quello che ci si attenderebbe da un sistema composto da moduli differenziati che sono tuttavia in grado di interagire efficacemente tra loro; in sostanza, la perturbazione iniziale mette in modo un sistema complesso (differenziato e integrato) che produce una risposta complessa. Le cose cambiano drammaticamente quanto lo stesso cervello, stimolato con gli stessi parametri (grazie al sistema di neuronavigazione), entra nel sonno NREM e perde coscienza. In questa condizione, osserviamo una forte risposta dell'area direttamente stimolata (a testimonianza del fatto che il cervello dormiente rimane attivo e re-attivo) che, tuttavia, non propaga alle altre zone della corteccia, rimanendo locale [27]. Il sistema talamocorticale, incosciente, è diventato un sistema modulare, un po' come la telecamera e il cervelletto. Non solo: la risposta dell'area stimolata è ora composta da un'onda semplice e se, sempre durante sonno NREM, provando a stimolare aree diverse otteniamo sempre la stessa risposta 
stereotipata. Al contrario, durante veglia, stimolazioni di aree diverse danno luogo a risposte elettriche molto differenziate. In conclusione, quando il sistema talamocorticale perde coscienza non solo perde l'integrazione, ma anche l'informazione [29] [30] [31]. In sintesi, le misure TMS/hd-EEG mostrano una differenza molto netta tra veglia e sonno, una differenza che è in accordo con le predizioni teoriche.

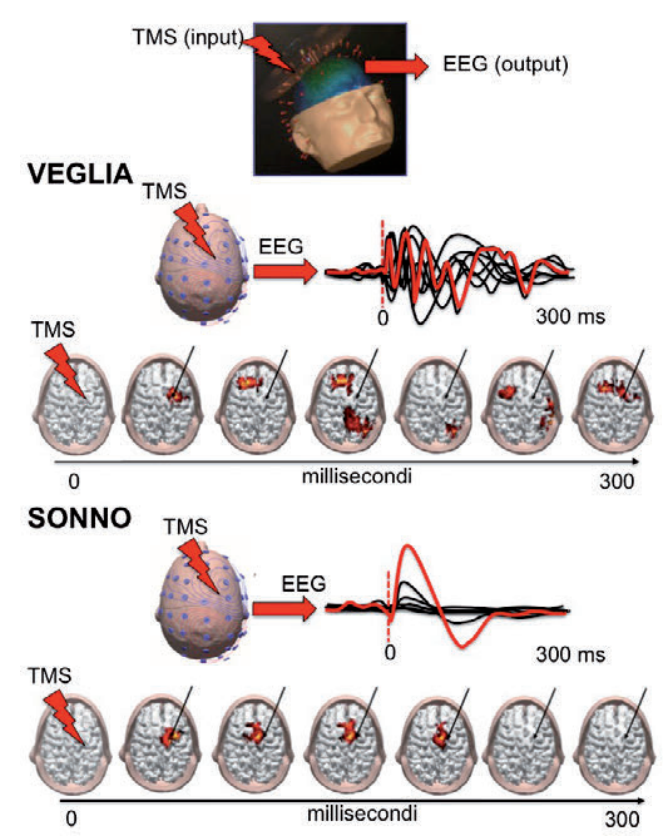

Fig. 4. Misure TMS/EEG durante sonno e veglia. Confronto tra le risposte EEG alla TMS ottenute durante veglia e sonno profondo (non-REM). Per ciascuna condizione, la saetta rossa indica il sito di stimolazione rispetto alla testa del paziente e alla posizione degli elettrodi (circoli blu sulla superficie dello scalpo). Le tracce a destra della testa rappresentano l'attività elettrica registrata da un sottoinsieme di elettrodi EEG dopo la perturbazione TMS (indicata dalla linea rossa tratteggiata). La traccia rossa si riferisce all'attività registrata dall'area di corteccia cerebrale posta immediatamente sotto lo stimolatore. Durante la veglia tutti gli elettrodi reagiscono alla perturbazione TMS e ognuno registra una risposta con caratteristiche specifiche. L'eco che ne risulta è complesso e diffonde a gran parte della corteccia cerebrale come illustrato dalle mappe di attivazione che "fotografano" a diversi istanti il propagarsi dell'attività neuronale. Al contrario, durante il sonno, la risposta alla perturbazione TMS è limitata all'elettrodo posto direttamente sotto lo stimolatore (traccia rossa) che registra un'onda stereotipata la quale non propaga alle altre aree cerebrali. L'eco è semplice e locale. Modificato da Massimini et al., Science 2005. 
Che cosa accade quando la coscienza riemerge nell'imprevedibile forma di un sogno nel cervello di un soggetto che giace immobile con gli occhi chiusi? Durante il sonno REM il sistema talamo-corticale, insieme alla coscienza, recupera la capacità di produrre risposte integrate e differenziate [32]. La TMS/hd-EEG svela in questa condizione il recupero di un complesso dialogo interno al cervello, nonostante il dialogo del soggetto con l'ambiente circostante sia momentaneamente abolito. Queste misure ci dicono che, in principio, possiamo svelare segni di coscienza anche in assenza di qualsiasi interazione del soggetto con il mondo esterno; chiaramente, questa possibilità sarà particolarmente rilevante quando ci troveremo davanti al letto di un paziente cerebroleso.

Le misure effettuate durante veglia, sonno NREM e sonno REM sembrano confermare la predizione teorica; tuttavia, se la teoria è corretta, la capacità del cervello di integrare informazione dovrebbe ridursi non solo quando la coscienza cambia in condizioni fisiologiche ma dovrebbe ridursi ogniqualvolta la coscienza viene ridotta; come, per esempio, durante anestesia generale. Non ci addentriamo nei dettagli a questo riguardo, se non per dire che recenti esperimenti [33] dimostrano che anche quando la coscienza è persa durante anestesia generale la risposta del cervello alla TMS è semplice e locale, molto simile a quella osservata durante sonno NREM. Questi risultati ci incoraggiano a portare le misure TMS/hd-EEG al letto del paziente in coma. Siamo infatti più vicini all'identificazione di un marker oggettivo della capacità del cervello di generare coscienza, che non dipende dalla capacità del soggetto di comprendere, comunicare o muoversi. Questo è uno degli obiettivi principali per far fronte alla necessità clinica ed etica di valutare correttamente, in modo sensibile e affidabile, il livello di coscienza nei pazienti portatori di gravi lesioni cerebrali.

\section{IL CERVELLO DOPO IL COMA}

Una volta al letto del paziente, ci possiamo chiedere prima di tutto come si presenta il pattern di risposta alla TMS in quei soggetti che si trovano in uno stato di coscienza minima [6] [7], ovvero in quei soggetti che hanno recuperato un certo grado di coscienza ma che, a causa della loro incapacità di comunicare, sono spesso confusi con i pazienti vegetativi. Una serie di misure recentemente condotte su un numero significativo di pazienti cerebrolesi dimostrano che la TMS/hd-EEG è invariabilmente 
in grado di distinguere i pazienti in stato di coscienza minima da quelli in stato vegetativo [34]. Nell'esempio riportato in Fig. 5 sono paragonati i pattern di attivazione cerebrale tipicamente riscontrati nelle diverse condizioni; mentre il cervello dei pazienti in stato vegetativo risponde come il cervello di un soggetto dormiente o anestetizzato, le risposte registrate nei pazienti in stato di coscienza minima sono diffuse e complesse.

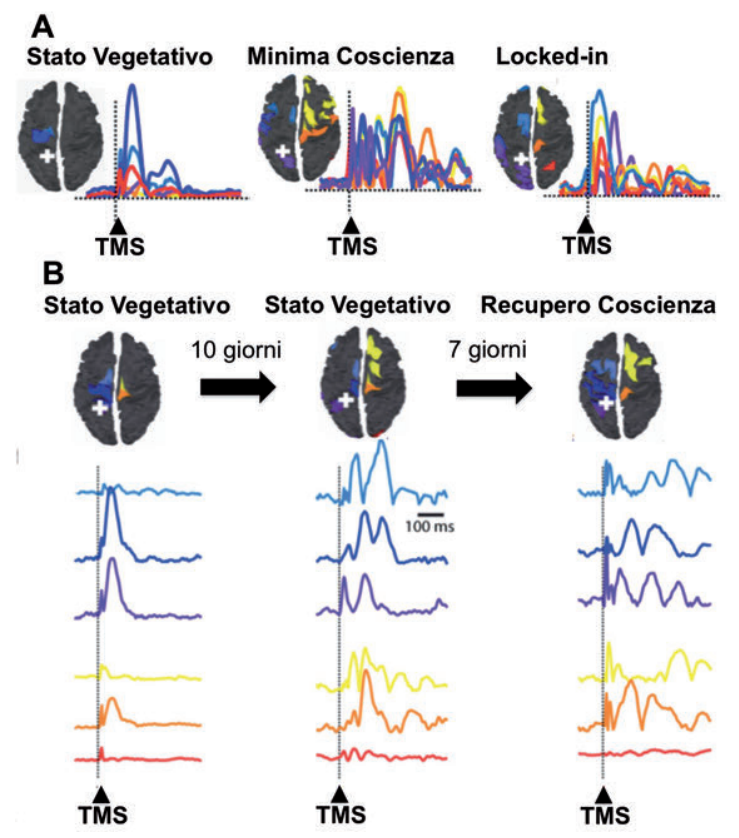

Fig. 5. Misure TMS/EEG di complessità nei pazienti. Il pannello A di questa figura illustra esempi tipici delle risposte ottenute in diverse categorie di pazienti (in stati vegetativo, in stato di minima coscienza e affetti da sindrome locked-in). La stimolazione di aree sane nel cervello di pazienti vegetativi induce sempre un'onda di attivazione semplice, simile a quella osservata durante sonno e anestesia. Nei pazienti locked-in, invece la risposta è invariabilmente indistinguibile da quella ottenuta in soggetti sani durante veglia. Sorprendentemente, lo stesso schema di attività complesso è osservato nel cervello di pazienti in stato di minima coscienza, a dispetto di segni clinici di coscienza che sono incerti e fluttuanti. Il pannello B riporta i risultati ottenuti in misurazioni ripetute eseguite in una paziente durante il recupero dal coma. La prima misurazione è condotta non appena la paziente esce dal coma ed entra nello stato vegetativo (occhi aperti). In questo caso, la riposta è ancora semplice e locale. Una misurazione effettuata 10 giorni dopo rivela uno schema di attività decisamente complesso, nonostante la paziente permanga dal punto di vista clinico in uno stato vegetativo. Sette giorni dopo, la paziente recupera coscienza ed è in grado di comunicare. Modificato da Rosanova et al., Brain 2012. 
Sorprendentemente, queste risposte non sono molto diverse da quelle che possiamo registrare in un soggetto sveglio o che sogna; sicuramente queste attivazioni sono simili a quelle registrate nei pazienti lockedin, i quali sono completamente paralizzati, ma pienamente coscienti.

Se la capacità delle diverse aree corticali di interagire tra loro (integrazione) ha effettivamente un ruolo causale nell'emergere di un barlume di coscienza, ci aspettiamo che un recupero del dialogo intracorticale sia chiaramente osservabile prima ancora che sia manifesta la capacità del paziente di comunicare con l'esterno. Infatti, Per provare che la misura è veramente sensibile dobbiamo riuscire a dimostrare che è possibile vedere la coscienza anche quando questa non dà il benché minimo segno di sé. Se il cannocchiale magnetico funziona davvero, dovrà essere capace di rilevare chiari segni di coscienza nel cervello prima che questa si appalesi attraverso il comportamento. Il pannello B della Fig. 5, mostra un caso paradigmatico a questo riguardo. Il caso è quello di una donna rimasta vittima di un grave trauma cranico (incidente stradale) a seguito del quale ha riportato varie lesioni a livello corticale e sottocorticale. Dopo l'incidente, la paziente rimane in coma per quattro settimane, e, in seguito si risveglia. Gli occhi sono aperti, il tono muscolare aumentato e sono presenti movimenti automatici del capo e degli arti, tuttavia, non è rivelabile alcun segno di coscienza. Si tratta di un recupero della vigilanza che non è accompagnato da un recupero della consapevolezza di sé e del mondo: il classico stato vegetativo. Eseguiamo la prima misura e registriamo la solita risposta semplice e locale, l'eco di un cervello in cui il delicato equilibrio della complessità e perduto. Dieci giorni dopo, la donna sembra per un attimo fissare e seguire la figlia nella stanza, ma questo rimane un evento isolato che non si ripete, forse un caso, e la diagnosi non cambia: stato vegetativo. Viene comunque effettuata eseguire un'altra misura con la TMS/EEG. Posizioniamo accuratamente la sonda tramite il sistema di neuro-navigazione e stimoliamo. Ciò che vediamo ci lascia, ancora una volta, meravigliati e perplessi: l'eco è indubbiamente quello di un cervello sveglio e cosciente! I risultati della TMS-EEG non collimano con il livello di coscienza stabilito clinicamente. In precedenza, avevamo osservato risposte complesse in individui che sembravano incoscienti, come i soggetti che sognavano e i pazienti locked-in; ma in tutti questi casi sapevamo già che la coscienza, per quando ben celata, era presente. Perché i soggetti lo avevano potuto raccontare: quelli che avevano sognato ci avevano in seguito riferito storie complicate e stravaganti, mentre i 
pazienti locked-in erano riusciti a segnalare chiaramente la loro piena consapevolezza grazie al faticoso codice binario dei movimenti oculari. In questo caso, non è presente alcun elemento che suggerisca la presenza di coscienza e non possiamo chiedere niente a nessuno. Eppure, il cervello sembra aver recuperato tutta la sua unità e tutta la sua ricchezza! Forse, questa volta, la sonda magnetica ha sbagliato di grosso o, forse, bisogna solo aspettare. Passano altri sette giorni, durante i quali la donna non dà alcun segno di sé, poi, improvvisamente, un chiaro segnale: fissa e segue con lo sguardo l'infermiera che si aggira nella stanza, apre la bocca come per dire qualcosa e alza il braccio destro, ripetutamente, verso il comodino. L'infermiera chiama i medici e che la trovano con la foto dei nipotini in mano e gli occhi lucidi. I neurologi la sottopongono immediatamente a tutti i test della coma recovery scale e la paziente risponde in modo riproducibile ai comandi con i movimenti appropriati, riconosce gli oggetti, muove il capo per dire sì o no e tenta di verbalizzare qualcosa che, tuttavia, è non ancora facilmente intelligibile. In pratica, la complessità era già emersa rivelando un universo nascosto che non era ancora riuscito a farsi riconoscere da chi stava fuori. Questo principio verrà regolarmente confermato negli anni successivi: in quasi il 20\% dei pazienti con diagnosi comportamentale di stato vegetativo, si trovano alti livelli di complessità cerebrale, che preannunciano la manifestazione di segni conclamati di coscienza [35].

\section{IMPLICAZIONI PRATICHE DI UNA MISURA DELLA COSCIENZA}

Definire una misura oggettiva per riconoscere la coscienza in chi non è in grado di scambiare informazione con l'ambiente esterno è impresa tutt'altro che facile. Come validarla se non possiamo sapere se il paziente è cosciente o meno? Un'equazione con due variabili, entrambe ignote, è ben difficile da risolvere. Gli esperimenti condotti fino ad ora indicano una via d'uscita. Infatti, le strade percorribili per spezzare questa circolarità sono due. La prima strada richiede di prendere in considerazione il fattore tempo e l'abbiamo appena descritta: possiamo seguire l'evoluzione dei pazienti che escono dal coma per verificare se la misura oggettiva di cui disponiamo anticipa il recupero della coscienza e della comunicazione a livello comportamentale. Se questo accade regolarmente, possiamo dire che la complessità è davvero la spia di una coscienza nascosta che non ha ancora trovato il modo di esprimersi al di fuori. La 
seconda strada prevede che la misura venga tarata usando come estremi della scala di riferimento condizioni in cui la coscienza è certamente presente (veglia, sogno) e condizioni in cui la coscienza è certamente assente (sonno profondo, anestesia generale). Poi, possiamo applicare la stessa misura a pazienti il cui stato è ignoto, o dubbio, per vedere dove si collocano in questa scala. Per fare questo, tuttavia, è necessario poter esprimere la complessità - che fino a questo punto abbiamo apprezzato solo in modo qualitativo - in termini quantitativi. La Fig. 6, illustra come ciò sia possibile. Basta "zippare" l'eco del cervello, proprio come si zippano le fotografie digitali che vogliamo inviare via email (il verbo to zip nel gergo informatico anglosassone significa comprimere).

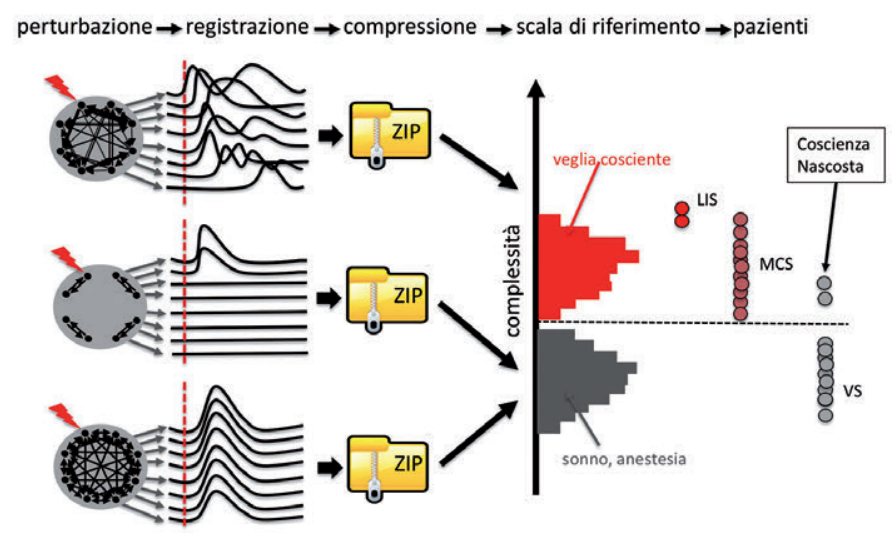

Fig. 6. Costruzione di una scala di riferimento. La parte sinistra della figura illustra il principio adottato per quantificare la complessità. L'eco cerebrale registrato dopo la perturbazione viene trattato come un'immagine bidimensionale e viene compresso utilizzando gli stessi algoritmi impiegati per "zippare" i file digitali. Quanto più l'eco è diffuso e differenziato, tanto meno sarà comprimibile e tanto più elevati saranno i valori di complessità. La parte destra della figura riporta i dati sperimentali. I valori di complessità ottenuti in presenza di coscienza (veglia) sono sempre più elevati di quelli ottenuti in condizioni in cui la coscienza è assente (sonno e anestesia). I pazienti locked-in (LIS) e quelli in stato di coscienza minima (MCS) hanno valori invariabilmente superiori al massimo valore riscontrato in assenza di coscienza (linea tratteggiata). I pazienti con diagnosi clinica di stato vegetativo (VS) che superano questa soglia sono da considerarsi coscienti.

Sappiamo tutti che più una foto digitale è complessa - tanto più è grande e tanto più diversi sono gli elementi che essa contiene - meno si riesce a comprimerla. Lo stesso principio generale si può applicare alle risposte cerebrali evocate dalla TMS [36]. Se l'eco evocato dalla pertur- 
bazione propaga a tutto il cervello e lo fa in modo molto differenziato da area ad area, sarà molto difficile da comprimere. Se, viceversa, l'eco rimane locale, o se propaga a tutto il cervello, ma lo fa con uno schema ripetitivo e ridondante, la risposta del cervello potrà essere facilmente compressa, usando pochi bit di informazione. In sintesi, il grado di compressibilità dell'eco - ossia il numero di bit necessari per descriverlo - fornisce una stima molto affidabile della complessità, un valore che possiamo porre sull'asse delle Y. Se, a questo punto, in un paziente che appare in tutto e per tutto in stato vegetativo troviamo un valore di complessità che si colloca in alto sull'asse della scala, dobbiamo assumere che egli è in qualche misura cosciente - contro ogni evidenza clinica e comportamentale. Va detto che questa la scala non costituisce un fine "coscienziometro", ma un metodo per cogliere un'eventuale capacità di coscienza anche nei casi in cui di coscienza non c'è il minimo segno.

Diagnosi a parte, andare direttamente al cuore del problema della coscienza può avere un'altra ricaduta pratica: misurare e capire i meccanismi della coscienza rappresenta un passo fondamentale se vogliamo promuoverne il recupero. Perché l'intero cervello, a seguito di una lesione più o meno localizzata, può rimanere bloccato in uno stato di bassa complessità per settimane, per mesi, o per sempre? Perché, a volte, la complessità ritorna improvvisamente, annunciando il riemergere di un soggetto cosciente? Come possiamo aiutare le aree di tessuto corticale rimaste integre a svegliarsi da questo strano sonno patologico? E poi, quanto cervello ci vuole per essere coscienti? Tutte domande destinate a rimanere, per il momento, senza una risposta definitiva. Tuttavia, alla luce di quanto detto finora, abbiamo il fondato sospetto che la coscienza nel cervello sia una questione di equilibrio, e che la perdita di questo equilibrio non sia necessariamente irreversibile. Basta chiudere i canali al potassio perché la massa informe di un cervello che dorme torni a essere la meravigliosa cattedrale del cervello cosciente. Ed è sufficiente ridurre un eccesso d'inibizione perché i moduli corticali di un cervello anestetizzato riprendano a dialogare intensamente tra loro e a integrare informazione. In molti pazienti che escono dal coma le lesioni strutturali sono così gravi - morte della maggior parte dei neuroni corticali o rottura della maggior parte degli assoni che li connettono - da non lasciare alcuna speranza, perché i margini di recupero strutturale del cervello sono piuttosto limitati. Ma negli altri pazienti? Nei molti pazienti in cui larghe porzioni del sistema talamocorticale rimangono intatte? Rimane il dubbio che alcuni cervelli siano bloccati 
e frammentati per via di un dettaglio funzionale, e che magari basti trovare la chiave perché un telaio inceppato ricominci a tessere le forme dell'esperienza soggettiva. Un dubbio per cui uno potrebbe decidere di dedicare la vita a capire come la complessità nasce, collassa e ritorna nella materia cervello.

\section{CONCLUSIONI}

All'inizio del viaggio abbiamo incontrato uno studente di medicina che, perplesso, soppesava il cervello di un altro uomo sul palmo della mano; poi, abbiamo sorvolato velocemente e molto superficialmente i campi della filosofia, della clinica, della neurofisiologia e delle neuroscienze teoriche per poi definire delle misure sperimentali molto grossolane e decisamente perfettibili. Cosa può dire il povero studente dopo questo tour-de-force? Sicuramente, dirà che la strada per capire la materia della coscienza è ancora molto lunga e difficile. Magari, penserà che qualcuno questa strada la deve pur intraprendere (a dispetto dello scetticismo), se non per altro, in nome dei pazienti. Allo studente potrebbe anche venire il sospetto che la materia molliccia che tiene in mano è, in fondo, veramente unica. Esiste un altro oggetto fisico così unitario e allo stesso tempo così ricco? Per un attimo, lo sfiora l'idea che, un giorno, a forza di raffinare teorie e misure, ci accorgeremo che non esiste nell'universo qualcosa di più grande del sistema talamocorticale di un essere umano.

\section{BIBLIOGRAFIA}

[1] Savoldi F, Ceroni M, Vanzago L. La Coscienza, ARAS, Fano, 2016.

[2] Epicuro, Opere, Einaudi, Torino, 1970, pagg. 62-63.

[3] Chalmers, D (1995). Il mistero dell'esperienza cosciente, in Cordeschi (a cura di), Filosofia della mente, Quaderni de Le Scienze, Milano 1996, 74-80.

[4] Jackson F (1986). Ciò che Mary non sapeva. In M. Salucci (a cura di), La teoria dell'identità. Alle origini della filosofia della mente, Le Monnier, Firenze, 166.

[5] Plum, F (1991) Coma and related global disturbances of the human conscious state. In: Normal and Altered States of Function Vol. 9, 359-425. Eds. A. Peters and E. G. Jones. Plenum Press, New York.

[6] Giacino JT, Ashwal S, Childs N, Cranford R, Jennett B, Katz DI, et al. (2002). The minimally conscious state: definition and diagnostic criteria. Neurology, 58, $349-53$. 
[7] Giacino JT, Kalmar K, Whyte J (2004). The JFK Coma Recovery Scale-Revised: measurement characteristics and diagnostic utility. Arch Phys Med Rehabil., 85, 2020-9.

[8] Plum F, \& Posner JB (1972). The diagnosis of stupor and coma. Contemporary Neurology Series, 10, 1-286. Royal College of Physicians (1994). Medical aspects of the persistent vegetative state. The Multi-Society Task Force on PVS. N Engl J Med., 330, 1499-508.

[9] Owen AM, Coleman MR, Boly M, Davis MH, Laureys S, \& Pickard JD (2008). Detecting awareness in the vegetative state. Annals of New York Academy of Sciences, 1129, 130-138.

[10] Boly M, Coleman MR, Davis MH, Hampshire A, Bor D, Moonen G, et al. (2007). When thoughts become action: An fMRI paradigm to study volitional brain activity in non-communicative brain injured patients. Neuroimage, 36, 979-992.

[11] Monti M, Coleman MR, \& Owen AM (2009). Neuroimaging and the vegetative state: Resolving the behavioral assessment dilemma? Annals of New York Academy of Sciences, 1157, 81-89.

[12] Majerus S, et al. (2005). Behavioral evaluation of consciousness in severe brain damage. Prog Brain Research, 150, 397-413. Review.

[13] Laureys S, Owen AM, \& Schiff ND (2004). Brain function in coma, vegetative state, and related disorders. Lancet Neurol, 3, 537-546.

[14] Steriade M, Timofeev I, \& Grenier F (2001). Natural waking and sleep states: a view from inside neocortical neurons. J Neurophysiol, 85, 1969-1985.

[15] Blumenfeld H, Westerveld M, Ostroff RB, Vanderhill SD, Freeman J, Necochea A, et al. (2003). Selective frontal, parietal, and temporal networks in generalized seizures. Neuroimage, 19, 1556-1566.

[16] Vanderwolf $\mathrm{CH}$ (2000). Are neocortical gamma waves related to consciousness? Brain Res, 855, 217-224.

[17] Tononi G, and Laureys S (2008). The neurology of Consciousness: an overview The neurology of Consciousness, Academic Press Inc., 375-411.

[18] Hobson JA, Pace-Schott EF, \& Stickgold R (2000). Dreaming and the brain: Toward a cognitive neuroscience of conscious states. The Behavioral and Brain Sciences, 23, 793-842.

[19] Tononi G (2004). An information integration theory of consciousness. BMC Neuroscience, 5, 42.

[20] Tononi G, Boly M, Massimini M, et al. Integrated information theory: from consciousness to its physical substrate. Nature Reviews Neuroscience. Volume: 17 Issue: 7 Pages: 450-461.

[21] Shannon CE and Weaver W (1963). The mathematical theory of communication. University of Illinois Press: Urbana.

[22] Sperry RW (1961). Cerebral organization and behavior: The split brain behaves in many respects like two separate brains, providing new research possibilities. Science. 2, 133, 1749-57.

[23] Bartels A, \& Zeki S (2005). The chronoarchitecture of the cerebral cortex. 
Philosophical Transactions of the Royal Society of London. Series B, Biological Sciences, 360, 733-750.

[24] Engel AK, Fries P, \& Singer W (2001). Dynamic predictions: Oscillations and synchrony in top-down processing. Nature Review, Neurosciences, 2, 704-716.

[25] Ilmoniemi RJ, Virtanen J, Ruohonen J, Karhu J, Aronen HJ, Naatanen R, et al. (1997). Neuronal responses to magnetic stimulation reveal cortical reactivity and connectivity. Neuroreport, 8, 3537-3540.

[26] Massimini M, Ferrarelli F, Huber R, Esser SK, Singh H, \& Tononi G (2005). Breakdown of cortical effective connectivity during sleep. Science, 309(5744), 2228-2232.

[27] Ferrarelli F, Massimini M, Sarasso S, Casali A, Riedner BA, Angelini G, et al. (2010). Breakdown in cortical effective connectivity during midazolam-induced loss of consciousness. Proc Natl Acad Sci U S A, 107(6), 2681-2686.

[28] Rosanova M, Casali A, Bellina, V, Resta F, Mariotti M, \& Massimini M (2009). Natural frequencies of human corticothalamic circuits. Journal of Neuroscience, 29, 7679-7685.

[29] Massimini M, Ferrarelli F, Esser SK, Riedner BA, Huber R, Murphy M, et al. (2007). Triggering sleep slow waves by transcranial magnetic stimulation. Proc Natl Acad Sci U S A, 104, 8496-8501.

[30] Massimini M, Boly M, Casali A, Rosanova M, \& Tononi G (2009). A perturbational approach for evaluating the brain's capacity for consciousness. Prog Brain Research, 177, 201-214.

[31] Tononi G, and Massimini M (2008). Why does consciousness fade in early sleep? Ann NY Acad Science 1129, 330-4.

[32] Massimini M, Ferrarelli F, Murphy M, Huber R, Riedner B, Casarotto S, et al. (2010). Cortical reactivity and effective connectivity during REM sleep in humans. Cogn Neurosci, 1, 176-183.

[33] Sarasso S, Boly M, Napolitani M, et al. (2015) Consciousness and Complexity during Unresponsiveness Induced by Propofol, Xenon, and Ketamine. Curr Biol. Dec 7;25(23):3099-105.

[34] Rosanova M, Grosseries O, et al. (2012). Recovery of cortical effective connectivity and recovery of consciousness in vegetative patients. Brain Apr;135:1308-20.

[35] Casarotto S, Comanducci A, Rosanova M, (2016). Stratification of unresponsive patients by an independently validated index of brain complexity. Ann Neurol. Nov;80(5):718-729.

[36] Casali AG, Gosseries O, Rosanova M, Boly M (2013). A theoretically based index of consciousness independent of sensory processing and behavior. Sci Transl Med. 2013 Aug 14;5(198):198. 